\title{
High Failure Rates of Concomitant Periprosthetic Joint Infection And Extensor Mechanism Disruption
}

\section{Authors:}

Lucas A. Anderson MD, Brian M. Culp MD, Craig J. Della Valle MD, Jeremy M. Gililland MD, R. Michael Meneghini MD, James A. Browne MD, Bryan D. Springer MD

Lucas A. Anderson MD ${ }^{\mathrm{a}, \mathrm{c}}$

Email: lucas.anderson@hsc.utah.edu

Brian M. Culp MD

Craig J. Della Valle MD ${ }^{b}$

Email: craigdv@yahoo.com

Jeremy M. Gililland MD ${ }^{\mathrm{c}}$

Email: Jeremy.Gililland@hsc.utah.edu

R. Michael Meneghini MD ${ }^{\mathrm{d}}$

Email:rm_meneghini@yahoo.com

James A. Browne MD

Email: JAB8HD@hscmail.mcc.virginia.edu

Bryan D. Springer MD ${ }^{\mathrm{a}} \quad$ [**Corresponding Author]

Phone: 704-323-2000

Fax: (704)323-3967

Email: Bryan.Springer@orthocarolina.com

(a) OrthoCarolina Hip and Knee Center

2001 Vail Avenue, Suite 200

Charlotte, NC 28207

(b) Orthopaedic Surgery at Rush University Medical Center

1611 West Harrison Street

Chicago, Illinois 60612

(c) University of Utah Department of Orthopaedics

590 Wakara Way

Salt Lake City, UT 84108

(d) Indiana University Department of Orthopaedics

340 West $10^{\text {th }}$ Street

Fairbanks Hall Suite 6200

Indianapolis, IN 46202

(e) University of Virginia Department of Orthopaedics

400 Ray C Hunt Dr \# 330,

Charlottesville, VA 2290 


\section{Abstract}

21 Introduction: Patients presenting with both chronic periprosthetic joint infection (PJI)

22 and extensor mechanism disruption (EMD) pose a significant challenge. As there is little

23 in the literature regarding outcomes of patients with concomitant PJI and EMD, we

24 performed a multicenter study to evaluate the outcomes.

25 Methods: Sixty patients with concomitant diagnoses of PJI and EMD were evaluated

26 from 5 institutions. Patient demographics, presentation type, surgical management and

27 outcomes including recurrent infections, final surgery and ambulatory status were

28 documented.

29 Results: Fifty-three of 60 patients had an attempted extensor mechanism

30 reconstruction/repair (EMR) of which $12(23 \%)$ were successful, averaging 3.5 (range 2-

31 7) intervening surgeries. Forty-one patients (77\%) were considered failures with

32 recurrence of infection as most common failure (80\%); 26 ended in fusion, 10 in above

33 knee amputation, 3 with chronic resection arthroplasty and 2 with chronic spacers/EMD.

34 Seven patients had no attempt at EMR but proceeded directly to fusion $(n=6)$ or

35 amputation $(n=1)$. There was no statistical difference between groups that had success or

36 failure of EMR in age, American Society for Anesthesiologists Physical Status

37 Classification System, or Body Mass Index.

38 Conclusions: Our study demonstrates that concomitant EMD and PJI is a dreaded

39 combination with poor outcomes regardless of treatment. Eradication of infection and

40 reconstruction of the extensor mechanism often requires numerous surgeries and despite

41 great effort often ends in failure. Consideration of early fusion or amputation may be

42 preferable in some patients to avoid the morbidity and mortality of repeated surgeries. 
43 Keywords: Infection, Extensor mechanism, fusion, amputation, failure

44 Level of Evidence: III

45

46 


\section{INTRODUCTION}

Infection following total knee arthroplasty (TKA) remains one of the most

49 dreaded and difficult complications to treat. The overall incidence of infection in the

50 literature ranges between $0.5 \%$ to $2 \%$ for primary TKAs and $2 \%$ to $4 \%$ for revision

51 TKAs.[1-4] In 2005, 16.8\% of all revision TKAs in the United States of America were

52 done because of infection and it is estimated that by the year 2030, $65 \%$ of all revision

53 procedures will be performed because of infection.[5] While successful eradication of

54 periprosthetic joint infection (PJI) has been reported in the range of $85-95 \%$, the mortality

55 associated with PJI is high.[6]

56 Disruption of the extensor mechanism is an infrequent, but catastrophic

57 complication following TKA. Reports in the literature range from 1.4 to 3.2 percent.[7-

58 10] Repair or reconstruction to the extensor mechanism disruption (EMD) is technically

59 challenging. Multiple techniques have been described and inconsistent results in the

60 literature with variable outcomes have been recorded.[11] A recent longitudinal study of

61 patients treated with extensor mechanism reconstruction (EMR) using allograft

62 demonstrated $69 \%$ of knees retained the allograft at a mean follow up of 68 months.

63 However, the reoperation rate was high at 58\% with the most common reason for

64 reoperation being development of PJI at 26\% .[12]

65 Patients presenting with both chronic PJI and EMD pose a significant challenge.

66 Both conditions are rare and the combination of the two diagnoses is even more rare.

67 Allograft or synthetic material used for reconstruction can create difficulty for infection

68 eradication. Removal of the extensor mechanism to treat the PJI can create substantial

69 functional disability. While in the past these complications were often treated with fusion, 
70 functional limitations associated with arthrodesis have led many to attempt

71 reconstruction/re-implantation in the hopes of maintaining greater function.[13, 14] To

72 date there is little data in the literature to guide surgeons and patients on the outcomes of

73 patients that end up with both of these devastating complications[12]. We performed a

74 multicenter study to evaluate the outcomes of patients that have concomitant PJI and

75 EMD. Our purpose was to evaluate the treatment strategies used and determine the

76 outcomes, including functional status, of patients that present with these complications.

77 In addition, we sought to determine risk factors for failure.

\section{METHODS}

We performed a multi-center retrospective review of patients with concomitant

82 tertiary referral centers' databases (XXX, XXX, XXX, XXX, XXX including 16

83 surgeons) was completed to identify all patients with a diagnosis of PJI (996.66) and

84 TKA removal (CPT 27385 and 27488) with keywords of Marlex, aortobifemoral,

85 quad(riceps) rupture, patella(r) tendon rupture and disruption. Patients less than 18 years

86 of age and native knees were excluded. Patients diagnosed with PJI and EMD but who

87 did not have an attempt at EMR but rather underwent early AKA or knee fusion were

88 included in the study for comparison of clinical outcome based upon number of surgeries,

89 complication rates, and ambulation status at final outcome. These cases were not

90 included in the "failure" rate of attempted extensor mechanism reconstruction.

91 Patient demographic data at time of index surgery for PJI/EMD was collected

92 retrospectively and included: age at the date of surgery, sex, body mass index (BMI), 
93 American Society for Anesthesiologists Physical Status Classification System (ASA

94 score). The timing of the primary TKA, diagnosis of infection, diagnosis of EMD,

95 infecting organism, and antibiotic resistance information was documented. Data was

96 collected regarding the presentation of PJI in relation to the timing of the EMD.

97 Additionally, presentation of PJI in relation to EMD was classified into the following

98 groups for ease of analysis: Group A: EMD occurred first and then PJI subsequently;

99 Group B: Concurrent EMD and PJI; Group C: PJI first and then EMD, thereafter. We

100 also recorded the type of EMR (primary repair or reconstruction with augmentation with

101 allograft, Marlex mesh, aortobifemoral endograft, etc.). If concurrent diagnoses of EMD

102 and PJI on presentation, then we also noted surgical management such as two-stage

103 exchange with EMR, arthrodesis, amputation, etc.

104 We documented presentation type, surgical management (i.e., two-stage exchange

105 with EMR, arthrodesis, amputation) and outcomes including reoperation (number of

106 operations to final outcome), recurrent infections, and final surgery and ambulatory

107 status. Ambulatory status was noted as yes/no; if yes (household or community) and

108 whether walking aide was required and what type (cane, crutches, walker, none).

109 We used the Musculoskeletal Infection Society (MSIS) diagnostic criteria [15].

110 This criteria defines that "PJI exists when either: There is a sinus tract communicating

111 with the prosthesis; or a pathogen is isolated by culture from at least two separate

112 samples obtained from the affected prosthetic joint; or three of the following five

113 criteria exist: 1) Elevated serum erythrocyte sedimentation rate (ESR) and serum C-

114 reactive protein (CRP) concentration, 2) Elevated synovial leukocyte count, 3) Elevated

115 synovial neutrophil percentage (PMN 4) Isolation of a microorganism in one culture of 
116 periprosthetic tissue or fluid, or 5) Greater than five neutrophils per high-power field in

117 five high-power fields observed from histologic analysis of periprosthetic tissue at $\times 400$

118 magnification."

119 Diagnostic criteria for extensor mechanism disruption included evidence on

120 clinical exam of extensor lag (> 15 degrees) against gravity or more and radiographic

121 evidence of a displaced patellar fracture disrupting the longtitudinal patella, patella alta or

122 patella baja. In some cases advanced imaging was utilized to diagnose EMD.

123 Criteria for successful extensor mechanism reconstruction included clinical

124 evidence of extensor mechanism continuity and function, which included continuously

125 palpated tissue and an extensor mechanism lag of 15 degrees or less against gravity.

126 Criteria for successful eradication of PJI was determined using the Delphi method

127 described by Diaz-Ledezma[16]. The consensus definition of a successfully treated PJI

128 is: (1) infection eradication, characterized by a healed wound without fistula, drainage,

129 or pain, and no infection recurrence caused by the same organism strain; (2) no

130 subsequent surgical intervention for infection after reimplantation surgery; and (3) no

131 occurrence of PJI-related mortality (by causes such as sepsis, necrotizing

132 fasciitis). Chronic antibiotic suppression was used in some cases as morbidity and

133 mortality of recurrent infection would not be tolerated by patient risk factors including

134 age and comorbidities and surgical history.

135 A total of 60 patients ( 22 men, 38 women) met the inclusion criteria. The mean 136 age of the cohort was 66 years (range 38-83; SD 9.4). The mean BMI was 34 (range 21137 49; SD 6.8). Overall, ASA score was II in 18, III in 27, IV in three patients, and missing 138 for 12. Of the 60 patients, 31 presented with EMD first and subsequently developed PJI 
139 (Group A), 17 patients presented with concurrent EMD and PJI (Group B), and 12

140 patients developed PJI first and then EMD later (Group C). Five of the 60 patients died

141 during the course of treatment.

\section{RESULTS}

143 Seven of the sixty patients were treated with early above the knee amputation

144 (AKA; $n=1)$ or knee fusion $(n=6)$ based upon comorbidities, soft tissue envelope, etc.

145 We included these for analysis of ambulation and number of surgeries, we did not include

146 them in analysis of failures of EMR as no attempt at reconstruction was made.

147 An attempt at EMR was made in 53 of the 60 patients. The types of EMR are

148 listed in Figure 1. Overall 12 of the 53 patients (23\%) had a successful reimplantation of

149 their TKA, defined as presence of a functional and continuous extensor mechanism and

150 no ongoing clinical evidence for PJI based on the defined criteria. The majority of those

151 with a successful outcome (7 of 12) had prior EMD and repair/reconstruction and

152 subsequently developed PJI (Group A) treated with a two-stage exchange reconstruction.

153 These patients underwent an average of 3.5 surgeries (range 2-7) between diagnosis and

154 last surgery.

155 Forty-one of fifty-three patients (77\%) were considered failures and averaged five

156 intervening surgeries (range 1-14). The primary mode of failure was recurrence of

157 infection in $80 \%$ of patients (33/41), 8 for failed extensor mechanism reconstruction

158 (20\%). Of the failures, 26 ended in fusion, 10 in AKA, 3 patients were left with chronic

159 extensor mechanism deficiency and two patients had retained chronic static spacers with

160 unresolved EMD.

161 
162

163

164

165

166

167

168

169

170

171

172

173

174 chronic antibiotics as treatment and two had retained chronic spacers.

Outcomes of Group B:

Seventeen patients were categorized into Group B, presenting with a concurrent

179 directly on to fusion and so were not included in failure analysis. The remaining 15

180 patients all underwent resection arthroplasty with placement of a static antibiotic spacer.

181 One patient was left with a chronic spacer in place with no further surgery.

182 Seven patients had an attempt at primary repair of the EMD at resection or re-

183 implantation. None of these were deemed to have a functioning extensor mechanism.

184 Five had a fusion, one an AKA and one was left with a chronic spacer after multiple 
185 irrigation and debridement's. Seven patients had attempted EMR at the time of

186 reimplantation with allograft or synthetic material. Three had successful retention of

187 TKA and functional EMR (two were maintained on chronic antibiotic suppression) and

188 four failed due to persistent infection resulting in four fusions and two AKAs.

190 Outcomes of Group C:

191 Twelve patients presented with a recent history of PJI and subsequent EMD. The

192 most common mechanism was a fall resulting in disruption of the patellar tendon. Eighty

193 percent failed due to recurrence of infection. 15\% had complete failure of the extensor

194 mechanism repair and 2 patients $(5 \%)$ had perarticular fractures resulting in need for

195 arthrodesis. The prior treatment of the PJI included eight patients that had undergone a

196 prior two-stage exchange with reimplantation and four patients that had an irrigation and

197 debridement with polyethylene exchange. One additional patient, not included in failure

198 analysis, had no attempt at repair and went directly to a knee fusion.

199 Of the 11 patients with EMD, 9 underwent EMR with either an allograft of

200 synthetic material. Two of these EMR were successful at regaining functional extensor

201 mechanism with minimal lag, no further infection, and required no additional surgery.

202 Seven patients had recurrence of infection and subsequently underwent an arthrodesis (4

203 patients) or an AKA (3 patient). Two patients underwent a primary repair of the EMD,

204 subsequent developed PJI, and had a resection and knee fusion.

205 We found no statistical associations with age, ASA, BMI or presenting category

206 (Group A, B, C) between the group of patients that had successful eradication of infection

207 and EMR versus those that failed either treatment of infection or had a failed EMR 
208 (Table 1). However, the failure group appeared to have a higher rate of infection with

209 resistant bacteria (MRSA) or polymicrobial infections.

210 Regarding functional status at latest follow-up of the 55 living patients (5 patients

211 died during course of treatment), 15 (27\%) of the patients are non-ambulators, 13 (24\%)

212 are homebound ambulators, and 27 (49\%) were community ambulators. Of the 15 non-

213 ambulators, 7 had an AKA, 6 had an arthrodesis, and 2 had an attempted EMR with

214 chronic spacer. All of the homebound ambulators required the use of a gait aid and 18 of

215 the 27 community ambulators required a gait aide. Only 9 of the 55 living patients in this

216 series required no walking aide at latest followup.

\section{DISCUSSION}

219 Extensor mechanism disruption in the setting of periprosthetic joint infection is a

220 rare but devastating combination. There is limited literature on this combination with

221 most reports focused on the treatment of one and only addressing the other as a noted

222 failure mechanism without details. The goal of infection management is to debride all

223 questionable tissue and leave no foreign material. Direct repair of EMD has poor results

224 and the bulk of the literature supports bringing in bulk allograft or synthetic tissue to

225 reinforce or bridge questionable native tissue. [17-19]

226 Patellar tendon rupture after TKA). Therefore, in the setting of concurrent PJI

227 and EMD, it is difficult to accomplish both goals in one surgical intervention.

228 Historically, the option that was considered best for these patients was arthrodesis as

229 recurrence of infection was thought to be high with the use of allograft reconstruction in 
230 the setting of prior infection.[14] The overall results and patient satisfaction with knee

231 arthrodesis are quite poor leading some to attempt EMR.[13]

233 presented with PJI and EMD to evaluate the treatment strategies used and determine

234 outcomes and functional status of patients that present with these complications. In

235 addition, we sought to identify risk factors for success and failure. Our study found that

236 of the 60 patients who met the inclusion criteria, over half (31 patients) presented with an

237 EMD first and then PJI developed subsequently with attempted treatment of the EMD

238 (Group A). This is not inconsistent with the prior literature of the ten major papers on

239 reconstruction of EMD.[7, 8, 10, 12, 20-23] These papers report on a total of 196 patients

240 and note that 12 were failures due to infection. Though treatment and outcomes were not

241 always delineated in these studies, approximately half of these failures were noted to

242 have had prior infection that had recurred and the other half appeared to have developed a

243 first time infection as a result of the EMD.

244 We found that the concurrent diagnoses of infection and EMD was rather morbid;

245 five of the 60 patients (12\%) in our series died during the course of treatment. This was

246 also found in several of the other sizable series (approximately 10\%-20\%).[20] Most of

247 the failures in the literature appeared to either be treated with AKA or fusion when noted

248 though several patients (similar to our cohort) were treated with chronic spacer retention,

249 antibiotic suppression and bracing.[21, 23]

250 While we expected that infection would lower rates of successful EMR we were

251 surprised that the success rate was as low as the $23 \%$ in our study. These patients also

252 clearly endured much as they underwent an average of 3.5 intervening surgeries to 
eradicate the infection and maintain a total knee with extensor function. $77 \%$ never did

254 have a successful EMR but still averaged five intervening surgeries. We found no

255 correlation between the type of surgery used to treat the infection and success or failure

256 likely due to sample size. The majority of the successful EMR in our series outcome were

257 from Group A (prior EMR and subsequently developed PJI) though this group was also

258 the largest group in our series. Success in this group was possibly due to the fact that

259 these knees already had a function extensor mechanism at the time of two-stage exchange

260 reconstruction and did not need augmentation of further tissue in face of infection.

261 When comparing function in patients treated with EMR, AKA and fusion, the

262 Knee Society Score and other outcome measures are not very valuable or equitable.

263 However, ambulation is a barometer of both the quality of life in many patients' eyes as

264 well as a reflection of independent function. We found that a third of our patients were

265 not able to regain any meaningful ambulation while half were community ambulators, the

266 majority of which needed some sort of walking aide. Fusion was the final surgical

267 outcome in the majority of our series (32 patients), which made up the majority of the

268 patients that were able to return to community ambulation. Only around half of the

269 patients in this series returned to community ambulation and only half of these were able

270 to ambulate without a walking aide.

271 Recurrence of infection was the most common mode of failure, re-occurring in

$27280 \%$ of attempts at joint salvage. While we hoped to identify patient characteristics

273 associated with failure that would direct the surgeon's treatment towards a discussion of

274 early fusion rather than reconstruction attempts, no such factors (age, ASA or BMI, etc.)

275 were found statistically significant. Instead, we found only a trend in infections with 
"resistant organisms" (MRSA, pseudomonas, and polymicrobial infections) seemed more

277 common in the patients that failed EMR.

This study has both strengths and a number of limitations. A multicenter study

279 allows us to pool together a larger group of patients with a very rare complication to

280 assess treatment trends and outcomes that might otherwise have not be possible with very

281 small numbers from a single institution. However, multicenter studies do involve

282 numerous surgeons with varying techniques and different decision making processes

283 when approaching a similar problem. In addition, there are inherent limitation with the

284 retrospective nature of this study in addition to the variability of patient presentations and

285 treatment outcomes. We are not able to make specific recommendations as to the optimal

286 treatment for patients presenting with PJI and EMD. The overall treatment outcomes

287 were poor and this study design allows us identify the overarching problem and focus on

288 the need for better treatment outcomes.

289 In conclusion, this study demonstrates that concomitant EMD and PJI is a

290 dreaded combination with poor outcomes regardless of treatment. Eradication of

291 infection and reconstruction of the extensor mechanism often requires numerous

292 surgeries and despite great effort ends in failure the majority of the time, usually due to

293 recurrent infection. Early consideration of fusion or amputation may be preferable to

294 avoid the morbidity and mortality of repeated surgeries. 


\section{REFERENCES}

297 [1] Della Valle, C., et al., American Academy of Orthopaedic Surgeons clinical practice guideline on: the diagnosis of periprosthetic joint infections of the hip and knee. Journal of Bone \& Joint Surgery - American Volume, 2011. 93(14): p. $1355-7$.

301 [2] Mihalko, W.M., et al., Infection in primary total knee arthroplasty: contributing factors. Instructional Course Lectures, 2008. 57: p. 317-25.

303 [3] Rand, J.A., B.F. Morrey, and R.S. Bryan, Management of the infected total joint 304 arthroplasty. Orthopedic Clinics of North America, 1984. 15(3): p. 491-504.

305 [4] Trousdale, R.T. and A.D. Hanssen, Infection after total knee arthroplasty. Instructional Course Lectures, 2001. 50: p. 409-14.

307 [5] Kurtz, S., et al., Projections of primary and revision hip and knee arthroplasty in 308 the United States from 2005 to 2030. J Bone Joint Surg Am, 2007. 89(4): p. 780-

310 [6] Berend, K.R., et al., Two-stage treatment of hip periprosthetic joint infection is 311 associated with a high rate of infection control but high mortality. Clinical 312 Orthopaedics and Related Research ${ }^{\circledR}, 2013.471(2):$ p. 510-518.

313 [7] Barrack, R.L., T. Stanley, and R.A. Butler, Treating extensor mechanism 314 disruption after total knee arthroplasty. Clinical orthopaedics and related 315 research, 2003. 416: p. 98-104.

316 [8] Burnett, R.S.J., et al., Extensor mechanism allograft reconstruction after total 317 knee arthroplasty. J Bone Joint Surg Am, 2004. 86(12): p. 2694-2699. 
318 [9] Emerson Jr, R.H., W.C. Head, and T.I. Malinin, Reconstruction of patellar tendon rupture after total knee arthroplasty with an extensor mechanism allograft. Clinical orthopaedics and related research, 1990. 260: p. 154-161.

LEOPOLD, M.S.S., et al., High Rate of Failure of Allograft Reconstruction of the Extensor Mechanism After Total Knee Arthroplasty*†. The Journal of Bone \& Joint Surgery, 1999. 81(11): p. 1574-9.

324 [11] Springer, B.D. and C.J. Della Valle, Extensor mechanism allograft reconstruction after total knee arthroplasty. The Journal of arthroplasty, 2008. 23(7): p. 35-38.

[12] Brown, N.M., et al., Extensor mechanism allograft reconstruction for extensor mechanism failure following total knee arthroplasty. J Bone Joint Surg Am, 2015. 97(4): p. 279-283.

[13] Gottfriedsen, T.B., H.M. Schrøder, and A. Odgaard, Knee Arthrodesis After Failure of Knee Arthroplasty. J Bone Joint Surg Am, 2016. 98(16): p. 1370-1377.

[14] Hasselberg, H., et al., Outcome of Knee Joint Arthrodesis as a Salvage Procedure After Periprosthetic Infection: An Analysis of 41 Patients. International Journal of Orthopaedics, 2016. 3(3): p. 571-576.

[15] Parvizi, J. and T. Gehrke, Definition of periprosthetic joint infection. The Journal of arthroplasty, 2014. 29(7): p. 1331.

336 [16] Diaz-Ledezma, C., C.A. Higuera, and J. Parvizi, Success after treatment of periprosthetic joint infection: a Delphi-based international multidisciplinary consensus. Clinical Orthopaedics and Related Research ${ }^{\circledR}, 2013.471(7)$ : p. 23742382. 
340 [17] Browne, J.A. and A.D. Hanssen, Reconstruction of patellar tendon disruption after total knee arthroplasty: results of a new technique utilizing synthetic mesh. Journal of Bone \& Joint Surgery - American Volume, 2011. 93(12): p. 1137-43.

[18] Cadambi, A. and G.A. Engh, Use of a semitendinosus tendon autogenous graft for rupture of the patellar ligament after total knee arthroplasty. A report of seven cases. Journal of Bone \& Joint Surgery - American Volume, 1992. 74(7): p. 9749.

347 [19] Rand, J.A., B.F. Morrey, and R.S. Bryan, Patellar tendon rupture after total knee arthroplasty. Clin Orthop Relat Res, 1989(244): p. 233-8.

349 [20] Emerson Jr, R.H., W.C. Head, and T.I. Malinin, Extensor mechanism reconstruction with an allograft after total knee arthroplasty. Clinical orthopaedics and related research, 1994. 303: p. 79-85.

352 [21] Nazarian, D.G. and R.E. Booth Jr, Extensor mechanism allografts in total knee arthroplasty. Clinical orthopaedics and related research, 1999. 367: p. 123-129.

[22] Browne, J.A. and A.D. Hanssen, Reconstruction of patellar tendon disruption after total knee arthroplasty. J Bone Joint Surg Am, 2011. 93(12): p. 1137-1143. after previous patellectomy. Clinical orthopaedics and related research, 2006. 450: 


\section{Figure Legend}

Figure 1: Attempted Extensor Mechanism Reconstruction Results 
Table 1: Factors Associated with Failure Versus Success

\begin{tabular}{|r|c|c|c|}
\hline & Re-implants \& EMR $(\mathrm{n}=12)$ & Fusion or AKA $(\mathrm{n}=48)$ & P-Value \\
\hline Age & 67 years (55-76 years) & 66 years $(38-83$ years $)$ & .91 \\
\hline ASA & & & \\
\hline & 0 & 0 & $.99 *$ \\
\hline I & 3 & 15 & \\
\hline II & 5 & 21 & \\
\hline III & 0 & 3 & .80 \\
\hline IV & $33(21-49)$ & $34(23-49)$ & \\
\hline
\end{tabular}

* The p-value was derived using a Fishers Exact Test. ASA was collapsed into two categories by combining ASA I and II versus III and IV. 


\section{Figure 1}

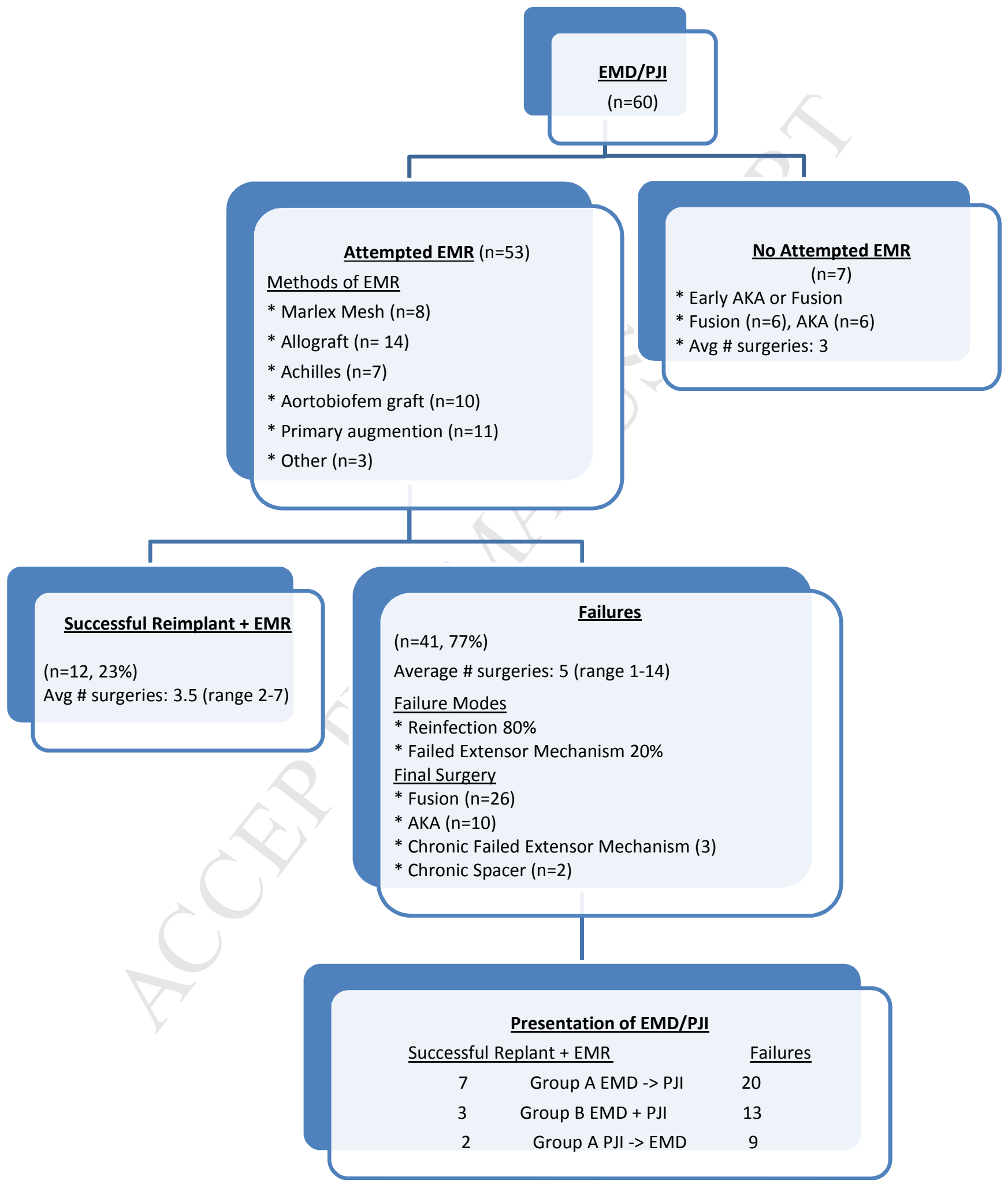

